\title{
Deep Neural Network for Breast Tumor Classification Through Histopathological Image
}

\author{
Amira Mofreh Ibraheem ${ }^{1 *}{ }^{[0000-0001-9831-2253]}$, Kamel Hussein Rahouma ${ }^{2,3}$, and \\ Hesham F. A. Hamed ${ }^{2,4}$ \\ ${ }^{1}$ School of engineering and applied science, Nile University, Giza, Egypt \\ ${ }^{2}$ Faculty of Engineering, Minia University, Minia, Egypt \\ ${ }^{3}$ Vice Dean of the Faculty of Computers, Nahda University in Beni Suef, Egypt \\ ${ }^{4}$ Faculty of Engineering, Egyptian - Russian University, Cairo, Egypt \\ *corresponding author E-mail: amofreh@nu.edu.eg
}

\begin{abstract}
Most oncologists differ in their opinions about the diagnosis using histopathological images, so the manual diagnosis of breast cancer is difficult, and it also needs high experience. Building a model of breast tumor classification is an essential task. Computer-aided diagnosis (CAD) enables efficient and accurate diagnosis of this type of imaging. Moreover, it helps early diagnosis of breast tumors. Deep learning has begun to evolve in the last decades, so convolutional neural networks $(\mathrm{CNN})$ are used to classify breast tumors. We introduced the automatic breast cancer classification methods to help doctors in examination and treatment, which can automatically classify the histopathological images as benign or malignant. This study's theoretical basis is developing a deep learning tumor classification system. It uses a deep learning approach, i.e., the proposed CNN Network consists of three stages. The first stage is preprocessing. The second stage is the feature extraction stage that takes input as augmented preprocessed images. This stage has 2 phases. The first phase consists of three parallel CNN branches with different filter sizes to extract different feature levels. We merged the three parallel branches in the second phase, which produced a feature fusion path that reduced the time complexity and enhanced the designed method's performance. The third stage classifies the fused features as benign or malignant. The BreakHis database is used and implemented and contains 7909 images of breast tumor tissue for 82 patients. The dataset was divided into 60\%, 20\%, 20\% for training, validation, and testing. Several tests were performed to achieve the best diagnostic accuracy of $91.37 \%$. The results are compared to the results of different techniques in the literature. The introduced advantages of our method make it help in the shortest treatment time, especially in the case of the large-scale histopathological images of the breast cancer dataset where the average testing time per image for the proposed method was $0.08 \mathrm{~s}$.
\end{abstract}

Keywords: Breast cancer, CAD system, Images classification, histopathology imaging, deep learning, convolutional neural networks, BreakHis database

\section{INTRODUCTION}

Many people lose their lives due to oncological diseases, the most dangerous of lung cancer and breast cancer [2]. Therefore, we need a computer-assisted system for the early detection and classification of these tumors to reduce mortality. This system must be rapid and highly accurate to help oncologists know the type of tumor [5] and to reduce the possibility of misdiagnosis [8]. There are different techniques for breast tumor images. One of the reliable types of images in diagnosing breast tumors is histopathology imaging. The oncologist examines a small tissue segment in a microscope and analyzes tissue structures, cell density, and shapes to know the tumor's location and its severity. This type of imaging needs excellent professional experience from oncologists, making the manual classification of pathological images of breast

cancer a significant challenge [10].

Revised:14 March, 2021, Accepted:9 May, 2021
Various histopathology imaging datasets such as the BreakHis dataset are taken from 82 women and contain 7,909 Images with four magnification factors 400x, 200x, 100x, and 40x [9].

Many researchers strive to provide much recent research concerning the diagnosis and classification of breast tumors with great accuracy. Nevertheless, It is still necessary to prepare a more accurate diagnostic CAD tool. A deep CNN model has been designed for efficient breast tumor classification that uses preprocessed histopathological images. We employed a new deep CNN model with multi-path feature extraction, which extracts features from preprocessed data. The proposed network consists of three stages: the preprocessing, the feature extraction stage, and the classification stage. The feature extraction stage contains three parallel paths. Each path has several layers consisting of convolutional, rectified linear units (ReLUs), and max-pooling. The extracted features are classified into two classes in the classification stage. 
CNN's main advantage is that it detects the important features automatically without any manual supervision. Classifying the images doesn't require feature selection. Also, it can operate with a $2 \mathrm{D}$ or $3 \mathrm{D}$ image dataset. CNN is also computationally efficient [12]. It employs convolution and pooling methods and offers parameter sharing. Connections can receive the same weight, diminishing the amount of processing data and reducing numerous parameters by removing those that are not important in the Feature Map, so the memory requirement is reduced. Since the number of parameters is drastically reduced, training time is proportionately reduced, which provides smoother and more reliable training.

The paper is organized as follows: Section 2 offers the proposed model implementation. Section 3 provides detailed information on testing environmental, database, and results. Lastly, Section 4 declares the conclusion.

\section{LITERATURE}

Lately, there are numerous methods for breast cancer diagnosis that used deep learning techniques that are available because of the significance of the breast cancer classification in histopathological images. S. Angara et al. demonstrate the ability of CNN to produce reliable predictions and evaluate classifier precision, specificity, precision, and sensitivity. The BreakHis database was used in this study at the image level. Their results were recorded for patient-level and patch levels. The dataset was divided into 5 validation folds and 5 training folds, which give an average accuracy of $88.35 \%$ in the patient-level case. The patch level case for all magnification factors gives an average accuracy of $87.94 \%$ [1]. In [3], Bayramoglu, $\mathrm{N}$. et al. presented two different $\mathrm{CNN}$ structures, a single task that used $\mathrm{CNN}$ to detect malignancy, which gives $83.25 \%$ accuracy, and a multi-task that used another $\mathrm{CNN}$ structure to detect both image magnification level and malignancy simultaneously, which gives $82.1 \%$ accuracy. The comparisons and the evaluations are carried out on all the magnification factors of the BreaKHis dataset.

Sudharshan, P. J., et al. in [4] presented a supervised learning system for breast cancer classification and explored the Multiple Instance Learning (MIL) relevance for the $\mathrm{CAD}$ system, depending on the patient's histopathological images analysis. BreakHis dataset is used for this study to classify the image for all magnification factors. The average accuracy is 82.67\%. In [6], Truong, T. and Pham, H. use the BreakHis dataset to test their simple CNN architecture, which contains several convolutional layers followed by fully connected layers, which achieved $77.3 \%$ average accuracy. M. Alrahhal [7] exploited the pretrained CNN network to detect breast cancer in histopathological images and gained an accuracy of $86.4 \%$ using the BraekHis database.
Golatkar, A. et al. [13] suggested a deep learningbased technique by fine-tuning the Inception-v3 network to classify breast images into four classes and obtain an average accuracy of $85 \%$. Rakhlin, A. et al. demonstrate a breast cancer classification approach that utilizes various DCNN with gradient boosted trees using ICIAR 2018 dataset. They reach $87.2 \%$ accuracy in the classification of the 4-classes [14]. Spanhol, F. A., et al. try to prove the BreakHis database efficiency for automated classification in two classes. The recorded accuracy ranges from $80 \%$ to $85 \%$ using different textural descriptors such as Completed LBP, Local Phase Quantization (LPQ), Grey-Level Cooccurrence, and the one keypoint descriptor [9].

$\mathrm{Li}$, Y. et al. [5] introduced a 4-class breast cancer classification for histological images using $\mathrm{CNN}$ and clustering algorithms and achieves $88.89 \%$ accuracy on the overall testing data. In [11], Zou, W. et al. classified the breast cancer image into two classes. Inception v1 convolutional neural network was used once with Spatial Pyramid Pooling (SPP)and again with a Global Average Pooling (GAP) to assure that images can be introduced in the original format. GAP gives an accuracy of $88.75 \%$ and an SPP accuracy of $89.25 \%$. More details about breast cancer classification systems can be found in [15-18].

\section{METHODOLOGY}

Fig. 1 presents an overview of our proposed system. There are three stages: preprocessing, feature extraction, and classification via CNN. Fig. 2 presents an overview of our network structure.

\subsection{Pre-processing}

The input images are resized [19] to 256x256x3, then the over-sampling and data augmentation methods are used to prevent overfitting and unbalanced class problems. The dataset is augmented by a two-level zoom range, $90^{\circ}$ rotation, and horizontal and vertical direction flip.

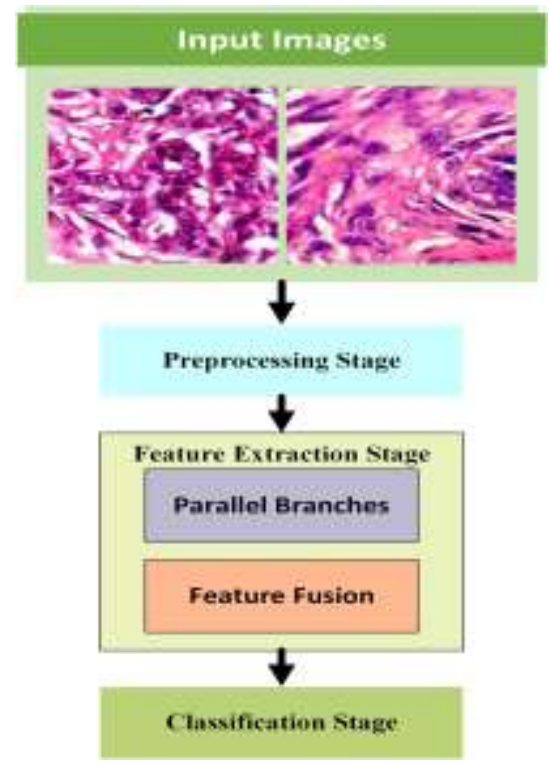

Fig. 1. The proposed system overall structure contains three main stages: preprocessing, feature extraction and 


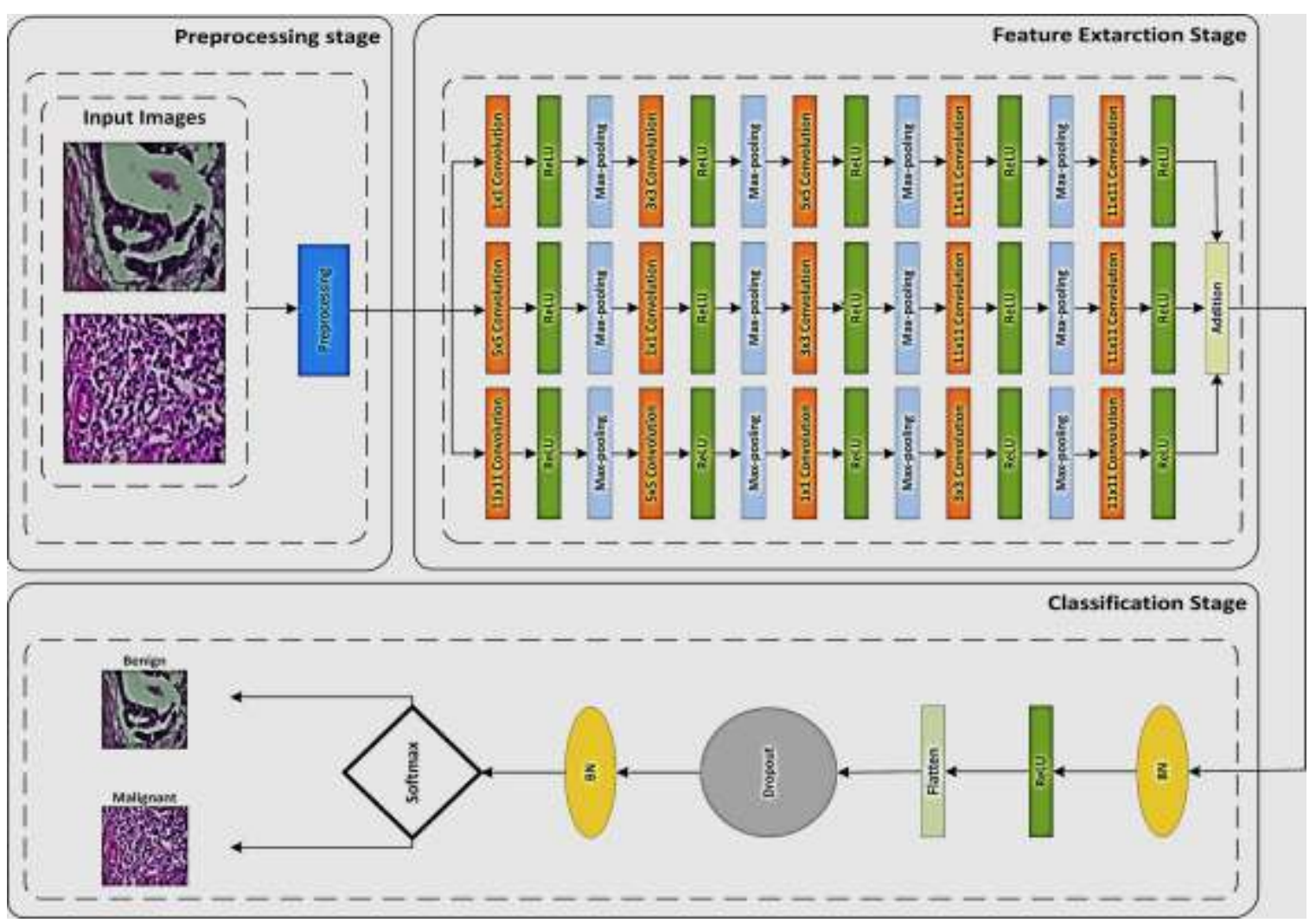

Fig. 2. The proposed CNN network overall structure.

\subsection{Feature extraction stage}

The feature extraction stage contains three parallel paths; each path consists of five convolutional layers and ReLU activation layers. Max-pooling layers are added after the convolutional layers for dimensionality reduction. The first layer in each line is a convolutional layer that has different filter sizes to extract different features level (low-level and highlevel). The Addition layer merges the three paths producing a single path with the sum of the extracted features (see Fig. 1).

\subsubsection{Convolutional Layers}

The main layer in $\mathrm{CNN}$ is the convolutional layer, which is used to extract features with a set of weights (filter) that convolves with the local regions of the input. Different filter sizes are used $1 \times 1,3 \times 3$, $5 \times 5$, or $11 \times 11$ to extract different feature levels. The stride of the hyper-parameter equals 1 , which is small compared with the filter size. Convolutional layers used 64 filters and applied rectified linear activation to local weights values.

\subsubsection{Rectified linear units (ReLUs)}

Nonlinearities are essential between layers to guarantee that the designed network is more significant than linear networks, which made it more powerful and comprises the ability to learn complex and complicated things from data [20]. The ReLU is the most activation function used in deep CNN applications. The main advantages are that it makes the network fast learning, gives better performance, and solves the vanishing gradient problem, which is represented in equation (1) [21].
AlgORITHM 1: BREAST TUMOR ClASSIFICATION METHOD THROUGH HistopatHOLOGICAL IMAGE

Input: Breast Histopathological RGB Image

Output: The CNN parameter, predicted table Reading the input image

Input Data $\leftarrow$ imread(folder)

Showing sample

imshow(Input Data )

Training:

for File in Listing do

Resize image dataset

Create a matrix to store all flattened images

Apply data augmentation by a two-level zoom range, $90^{\circ}$ rotation, and horizontal and vertical direction flip

Initialize hypermeters such as batch size, the number of epochs, the number of filters, kernel size, number of classes.

Split the augmented dataset into training and testing set.

Generate binary class matrices of the class vectors

Define proposed CNN architecture

Train the defined architecture

fine-tuned hyperparameters to choose the best model and avoid over-fitting

save weights

end for

Testing:

For I in range ( ) do

Re-define CNN architecture and Load weights

Test and validate the proposed architecture

Classify the classes and generate confusion matrix

Calculate Sensitivity, Specificity, and accuracy

Visualize Sensitivity, Specificity, and accuracy. save weights

End for 

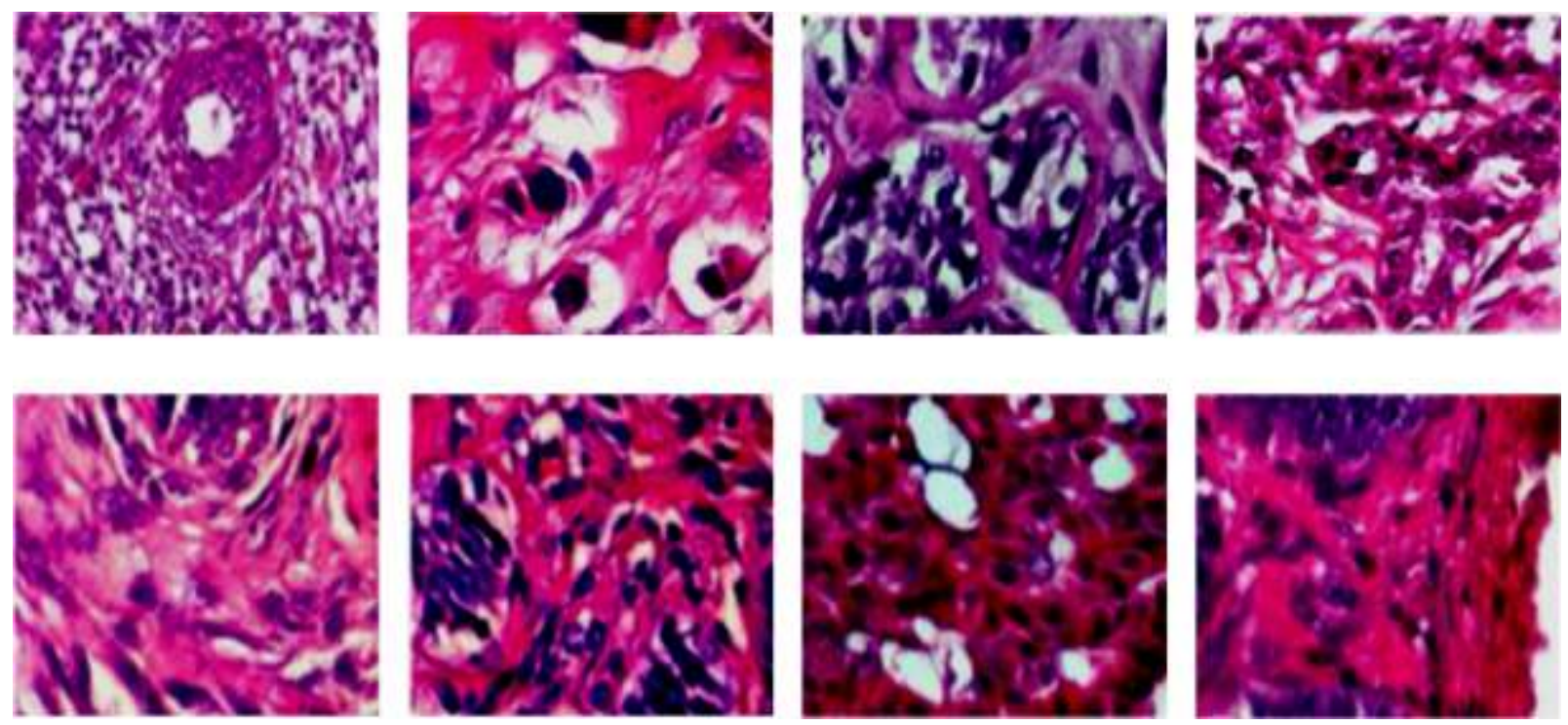

Fig. 3. Several types of malignant and benign tumors of the BreaKHis histopathological images database with a 400x magnification factor.

\section{3eLU $(Y)=\max (0, Y)$ \\ 3.2.3 Pooling layer}

The max-pooling layer is applied to minimize the feature map by reducing similar features. The noise drop, dimension reduction, and receptive field amplification are the main purpose of max-pooling layers [22]. The network uses max-pooling with a stride of 2 and size $3 \times 3$.

\subsubsection{Addition layer}

Layer that adds a number of input features. It takes the output features of the three parallel CNN's networks, all of the same shape, and returns a single value.

\subsection{Classification stage}

The classification stage that classifies the merged features into two classes for four magnification factors contains different layers: batch normalization $(\mathrm{BN})$, $\mathrm{ReLu}$, flatten, Dropout, and dense layer with softmax activation function as classification layer (see Fig. 1).

\subsubsection{Batch normalization (BN)}

$\mathrm{BN}$ is a useful regularizer that improved generalization and additionally accelerated convergence. It is capable of dealing with an inward covariate shift issue. Also, there is a problem that must be considered, the changing of circulation of each data source layer in the training phase, which changes weights for each layer, which changes the output activation distribution and slows training. Therefore, $\mathrm{BN}$ is used to overcome this problem [20].

\subsubsection{Flatten layer}

It is utilized to convert the input to a flat vector output without affecting the size of batches.

\subsubsection{Dropout layer}

Dropout is an effective regularization method that randomly rejects the selected neurons and stops their weights from updating during training [22].

\subsubsection{Softmax}

The output activation function is softmax, according to equation (2):

$$
W_{j}=\frac{\exp \left(X_{j}\right)}{\sum_{i=1}^{n} \exp \left(a_{i}\right)}
$$

where $X$ is the input, and $W$ is the output with $n$ neurons. The denominator $\sum_{i=1}^{n} \exp \left(X_{i}\right)$ normalizes the output to ensure that $\sum_{i=1}^{n} W_{j}=1$ and $0 \leq W_{j} \leq 1$, which can be graphically showed as an X layer with $n$ neurons. The final layer is the classification layer that returns the classification output of the neural network. The categorical cross-entropy loss (error) is applied for multi-class classification [23].

\section{TESTING ENVIRONMENT}

This section explains the experiment's environment and the BreaKHis database, which is used to evaluate the proposed method.

\subsection{Machine tool}

The experimental work applied to the Jupiter notebook was used by Keras, Tensor-Flow, and python toolkits. We equipped the computer with $24 \mathrm{~GB}$ of RAM and a Core 17 processor, which runs at $3.2 \mathrm{GHz}$.

\subsection{Dataset}

The proposed method is tested by a Histopathological Breast Cancer Image Classification database [9] (see Fig. 3). BreakHis dataset was implemented in P\&D Laboratory, Brazil. It takes by surgical open biopsy (SOB), ready for the histological survey, and manually labeled by expert oncologists. It has taken from 82 women suffering from a breast tumor to collect 7909 RGB images in nature size $760 \times$ 460 pixels. It contains different types of benign and malignant tumors; each type has four magnification factors. Table 1 provides detailed information on the distribution of the different types [9]. We divide the dataset into 1593 testing images, 1,263 validation images, and 5,053 training images.

\section{Results and Discussion}




\section{Vol.42, No.1. January2023}

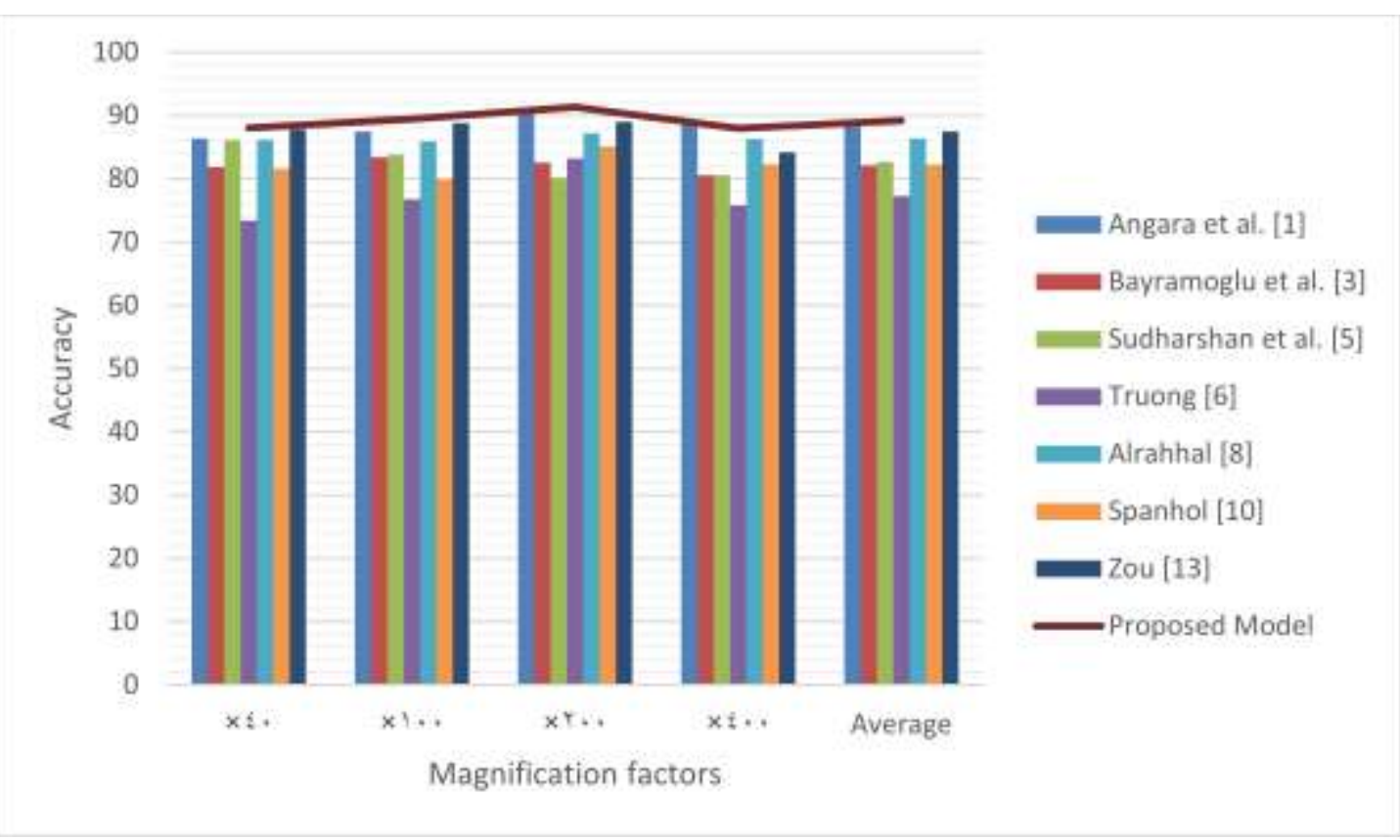

Fig. 4. Comparison between the proposed method and the literature of the classification accuracy with different magnification factor.

\subsection{Evaluation metrics}

Table 1. BreakHis dataset image distribution for different magnification factor and various subtypes

\begin{tabular}{|c|c|c|c|c|c|}
\hline \multirow{2}{*}{$\begin{array}{l}\text { Tumor } \\
\text { type }\end{array}$} & \multirow{2}{*}{ Subclass } & \multicolumn{4}{|c|}{ Magnification Factors } \\
\hline & & $400 X$ & $200 X$ & $100 X$ & $40 X$ \\
\hline \multirow{4}{*}{.0.0 } & $\begin{array}{l}\text { Phyllodes } \\
\text { Tumor }\end{array}$ & 130 & 140 & 150 & 149 \\
\hline & $\begin{array}{l}\text { Tubular } \\
\text { Adenoma }\end{array}$ & 115 & 108 & 121 & 109 \\
\hline & Fibroadenoma & 237 & 264 & 260 & 253 \\
\hline & Adenosis & 106 & 111 & 113 & 114 \\
\hline \multirow{4}{*}{ 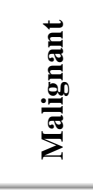 } & Papillary & 138 & 135 & 142 & 145 \\
\hline & Mucinous & 169 & 196 & 222 & 205 \\
\hline & Lobular & 137 & 163 & 170 & 156 \\
\hline & Ductal & 788 & 896 & 903 & 864 \\
\hline \multicolumn{2}{|c|}{$\begin{array}{l}\text { Total images } \\
\text { (magnification) }\end{array}$} & 1820 & 2013 & 2081 & 1995 \\
\hline \multicolumn{2}{|c|}{$\begin{array}{l}\text { Total images } \\
\text { (82 patient) }\end{array}$} & \multicolumn{4}{|c|}{7909} \\
\hline
\end{tabular}

In order to test and evaluate the proposed system and ensure its effectiveness, reliable standards such as accuracy are utilized. The results were taken across the patch levels, not the patient level. The confusion matrix's parameters are essential to computing the Sensitivity, Specificity, and Accuracy. The mathematical equation (3) shows the accuracy $[24,25]$.

$$
\text { accuracy }=\frac{T_{\text {true }}}{T}
$$

where $T_{\text {true }}=\Psi+\eta$ is the total true cases, and $\mathrm{T}$ is the total number of cases.

$$
\begin{aligned}
& \text { Sensitivity }=\frac{(\Psi)}{\left(T_{P}\right)} \\
& \text { Specificity }=\frac{(\eta)}{\left(T_{N}\right)}
\end{aligned}
$$

where true positives $(\Psi)$ is the correctly classified positive cases, true negatives $(\eta)$ is the correctly classified negative cases, false negatives $(\omega)$ is the wrongly classified negative cases, false positives $(\beta)$ is the wrongly classified positives cases, total positives cases $\left(T_{P}\right)$ is the total classified positive cases, and total negatives cases $\left(T_{N}\right)$ is the total classified negative cases.

$$
\text { confusion matrix }=\left(\begin{array}{ll}
\Psi & \omega \\
\beta & \eta
\end{array}\right)
$$

Matthew Correlation Coefficient (MCC) is the way of computing the Pearson product-moment correlation coefficient between predicted and actual values, which is unaffected by the dataset's unbalanced issue [26].

$$
M C C=\frac{\Psi \cdot \eta-\beta \cdot \omega}{\sqrt{(\Psi+\beta)(\Psi+\omega)(\eta+\beta)(\eta+\omega)}}
$$

\subsection{Results}

This section discusses the outcomes of the demonstrated system in fulfilling the breast cancer classification task through several experiments. To further characterize performance, confusion matrix analyses were conducted. The confusion matrices of the 2-class patch classification of the four magnification factors, accuracy, sensitivity, and specificity presented in Table 2 , proved that our system could classify the confusion correctly. The experiments illustrate that the proposed network gained better performance in accuracy than other literature approaches (see Fig. 4), which represents that 


\section{Vol.42, No.1. January2023}

the presented network can avoid overfitting and generalization. Tables 3 presents a comparison with different literature methods. The proposed model has achieved the best accuracy of $91.37 \%$ at the 200x magnification factor and an average accuracy of $89.22 \%$. Fig. 5 shows some experimental results of different tumor images with 200x magnification histopathological images, as confirmed in the result section. This proposed model can be applied in hospitals suffering from a severe shortage in the number and experience of professional oncologists with multiple classifications of breast cancer. In the case of the large-scale histopathological images of the breast cancer dataset, the oncologist will waste much
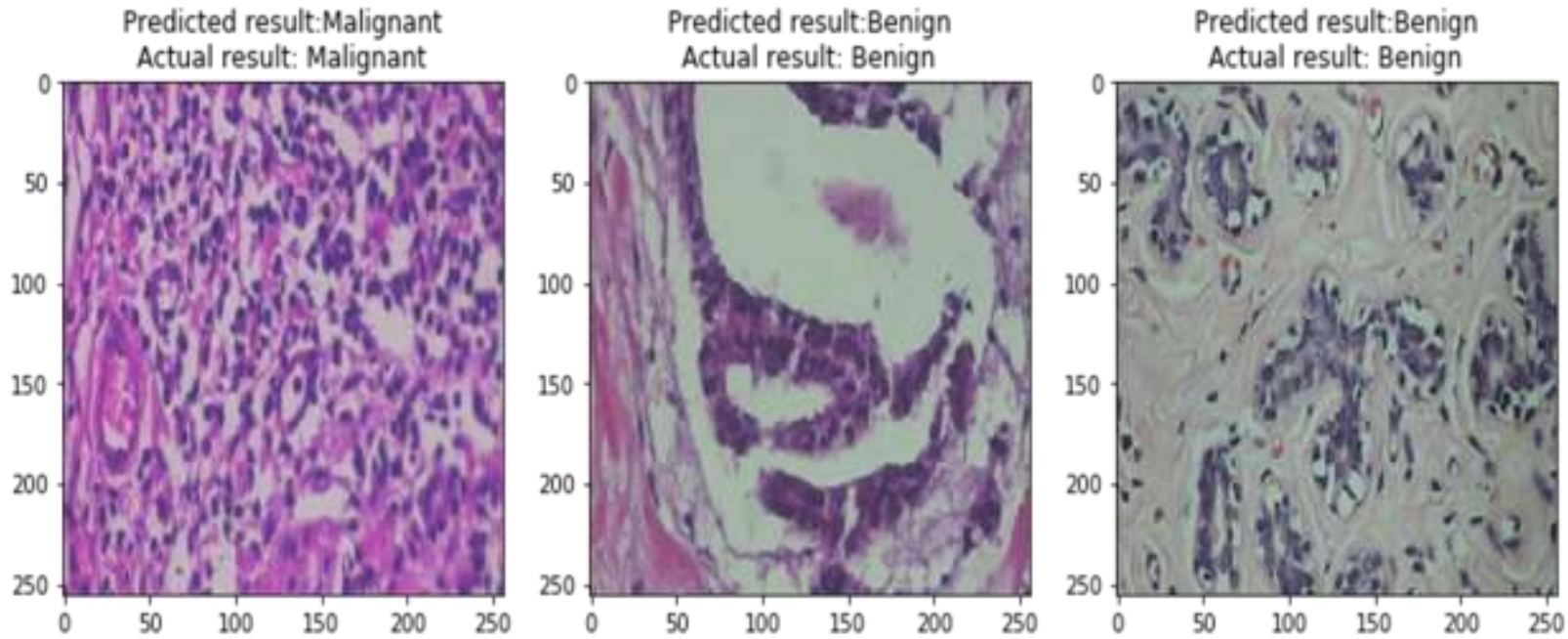

Fig. 5. Several experimental results of malignant and benign tumors of the BreaKHis histopathological images database with a 200x

\begin{tabular}{lcccc} 
Accuracy & $\mathbf{8 8 . 0 2}$ & $\mathbf{8 9 . 4 9}$ & $\mathbf{9 1 . 3 7}$ & 88 \\
Sensitivity & 92.73 & 95.85 & 100 & 93.55 \\
Specificity & 77.77 & 75.38 & 72.22 & 76.47 \\
MCC & 71.82 & 74.89 & 80.12 & 72.12 \\
\hline
\end{tabular}

Table 3. Comparison between the proposed method and the literature of the classification accuracy (\%) on four Magnification factors in image level. The best mean results in different Magnification factors are in bold

\begin{tabular}{llllll}
\hline \multirow{2}{*}{ Method } & \multicolumn{4}{c}{ Magnification factors $(\boldsymbol{\%})$} & Average \\
\cline { 2 - 5 } & $\mathbf{4 0 \times}$ & $\mathbf{1 0 0 \times}$ & $\mathbf{2 0 0 \times}$ & $\mathbf{4 0 0 \times}$ & $\mathbf{( \% )}$ \\
\hline$[1]$ & 86.4 & 87.5 & 90.5 & $\mathbf{8 9}$ & 88.35 \\
{$[3]$} & 81.87 & 83.39 & 82.56 & 80.6 & 82.1 \\
{$[4]$} & 86.1 & 83.8 & 80.2 & 80.6 & 82.67 \\
{$[6]$} & 73.41 & 76.77 & 83.22 & 75.81 & 77.3 \\
{$[7]$} & 86.2 & 85.9 & 87.2 & 86.3 & 86.4 \\
{$[9]$} & 81.6 & 79.9 & 85.1 & 82.3 & 82.22 \\
{$[11]$} & 87.81 & 88.81 & 89 & 84.17 & 87.44 \\
Proposed & $\mathbf{8 8 . 0 2}$ & $\mathbf{8 9 . 4 9}$ & $\mathbf{9 1 . 3 7}$ & 88 & $\mathbf{8 9 . 2 2}$ \\
\hline Model & & & & & \\
\hline
\end{tabular}

factors.

\subsection{Discussion}

The proposed model achieved a reliable and accurate recognition rate. Although the classification process is complicated due to the presence of very fine-grained, the proposed method is stable for different Magnification factors. It has the highest accuracy in the classification of breast cancer through effort and time, which will increase the labeling errors.

It is a massive advantage of the proposed method for whole slide images (WSI) classification to keep full global information about breast cancer images and avoid limitations of correction methods. Although correction methods are standard, they have a drawback as the cancer area is only a small portion of the histopathological images of breast cancer, leading to a diagnostic error by the oncologist. Therefore, WSI is being used carefully as model inputs to reduce oncologists' workload and improve diagnostic efficiency.

\subsubsection{Workflow overview}

The workflow can be understood into three main stages. The explanation of the stages is provided as follows:

\section{- Training stage}

It learns sufficient feature representation and optimizes the different classes' feature space distance. The proposed method learns the high-level feature maps and the hierarchical features in the first stage, then enters the addition layer, BN normalizations, and Relu. The outputs of the two stages are flattened and transmitted to minimize the intra-class distance and maximize the interclass Euclidean distance. Finally, the Adam optimizer optimizes the categorical crossentropy losses.

\section{- Validation stage}

It is fine-tuned hyperparameters to choose the best model and avoid over-fitting.

\section{- Testing stage}

It is used to get the proposed method performance. 


\subsection{2 augmentation}

Augmentation is the way to create a new or enlarge dataset from an existing dataset without gathering new dataset samples. Domain-specific techniques can be applied to samples from the dataset, which generates new samples. Training CNN networks with more datasets led to more skillful networks. Also, the augmentation provides variations of images that increase the network's ability to generalize. The augmentation techniques as flipping, padding, and cropping are usually used to train prominent $\mathrm{CNN}$. Over-sampling and augmentation are applied in the proposed system to avoid unbalanced problems and over-fitting. The used augmentation techniques are:

1. Zoom range with 3 .

2. Rotation at $90^{\circ}$.

3. Horizontal and vertical flipping.

\section{Conclusion and future work}

In this study, we design a new CNN for the Breast Cancer Classification for Histopathology Image. The design contains three stages; the first stage is the preprocessing; then, the second stage is the feature extraction from preprocessed input images, lastly, classifying these features as 2-class classification in the classification stage. The model has been tested using the BreakHis dataset and achieved a promising result on the magnification factor of 200x; the maximum accuracy recorded $91.37 \%$. Our method has been compared with other methods mentioned in state of the art. The focal points in the future will be developed to multi-class classification, and we aspire to find an optimal way to predict the breast tumor.

\section{References}

[1] S. Angara, M. Robinson, and P. Guillén-Rondon, "Convolutional Neural Networks for Breast Cancer Histopathological Image Classification," in 2018 4th International Conference on Big Data and Information Analytics (BigDIA), 2018, pp. 1-6.

[2] N. Christian. (2018, 19/2/2020). What to know about breast cancer. Available:

https://www.medicalnewstoday.com/a rticles/37136

[3] N. Bayramoglu, J. Kannala, and J. Heikkilä, "Deep learning for magnification independent breast cancer histopathology image classification," in 2016 23rd International Conference on Pattern Recognition (ICPR), 2016, pp. 24402445.

[4] P. J. Sudharshan, C. Petitjean, F. Spanhol, L. E. Oliveira, L. Heutte, and P. Honeine, "Multiple instance learning for histopathological breast cancer image classification," Expert Systems with Applications, vol. 117, pp. 103-111, 2019/03/01/ 2019.

[5] Y. Li, J. Wu, and Q. Wu, "Classification of Breast Cancer Histology Images Using Multi-Size and Discriminative Patches Based on Deep Learning," IEEE Access, vol. 7, pp. 21400-21408, 2019.

[6] T. D. Truong and H. T.-T. Pham, "Breast Cancer Histopathological Image Classification Utilizing Convolutional Neural Network," in 7th International Conference on the Development of Biomedical Engineering in Vietnam (BME7), Singapore, 2020, pp. 531-536: Springer Singapore.

[7] M. Alrahhal, "Breast Cancer Classification in Histopathological Images using Convolutional Neural Network," International Journal of Advanced Computer Science and Applications, vol. 9, 04/01 2018.

[8] D. Shen, G. Wu, and H.-I. Suk, "Deep Learning in Medical Image Analysis," (in eng), Annual review of biomedical engineering, vol. 19, pp. 221-248, 2017.

[9] F. A. Spanhol, L. S. Oliveira, C. Petitjean, and L. Heutte, "A Dataset for Breast Cancer Histopathological Image Classification," IEEE Transactions on Biomedical Engineering, vol. 63, no. 7, pp. 14551462, 2016. 


\section{Vol.42, No.1. January2023}

[10] L. He, L. R. Long, S. Antani, and G. R. Thoma, "Histology image analysis for carcinoma detection and grading," Computer Methods and Programs in Biomedicine, vol. 107, no. 3, pp. 538556, 2012/09/01/ 2012.

[11] W. Zou, H. Lu, K. Yan, and M. Ye, "Breast Cancer Histopathological Image Classification using Deep Learning," in 2019 10th International Conference on Information Technology in Medicine and Education (ITME), 2019, pp. 53-57.

[12] L. Alzubaidi et al., "Review of deep learning: concepts, $\mathrm{CNN}$ architectures, challenges, applications, future directions," Journal of Big Data, vol. 8, no. 1, p. 53, 2021/03/31 2021.

[13] A. Golatkar, D. Anand, and A. Sethi, "Classification of Breast Cancer Histology Using Deep Learning," Cham, 2018, pp. 837-844: Springer International Publishing.

[14] A. Rakhlin, A. Shvets, V. Iglovikov, and A. Kalinin, "Deep Convolutional Neural Networks for Breast Cancer Histology Image Analysis," 02/02 2018.

[15] M. Veta, J. P. W. Pluim, P. J. v. Diest, and M. A. Viergever, "Breast Cancer Histopathology Image Analysis: A Review," IEEE Transactions on Biomedical Engineering, vol. 61, no.

5, pp. 1400-1411, 2014.

[16] V. Gupta and A. Bhavsar, "Breast Cancer Histopathological Image Classification: Is Magnification Important?," in 2017 IEEE Conference on Computer Vision and Pattern Recognition Workshops (CVPRW), 2017, pp. 769-776.

[17] M. A. Aswathy and M. Jagannath, "Detection of breast cancer on digital histopathology images: Present status and future possibilities," Informatics in Medicine Unlocked, vol. 8, pp. 7479, 2017/01/01/ 2017.

[18] A. Nahid and Y. Kong, "Involvement of Machine Learning for Breast Cancer Image Classification: A Survey," Computational and Mathematical Methods in Medicine, vol. 2017, pp. 1-29, 12/31 2017.

[19] D. Hassanin, m. Abdellah, A. Khalaf, and R. R. Gharrieb, "Automatic localization of Common Carotid Artery in ultrasound images using Deep Learning," (in en), Journal of Advanced Engineering Trends, vol. 40, no. 2, pp. 127-135, 2021.

[20] M. Abd-Ellah, A. I. Awad, A. A. M. Khalaf, and H. Hamed, "Deep Convolutional Neural Networks: Foundations and Applications in Medical Imaging," 2020, pp. 233-260.

[21] M. K. Abd-Ellah, A. I. Awad, A. A. M. Khalaf, and H. F. A. Hamed, "Two-phase multi-model automatic brain tumour diagnosis system from magnetic resonance images using convolutional neural networks," EURASIP Journal on Image and Video Processing, vol. 2018, no. 1, p. 97, 2018/09/30 2018.

[22] M. K. Abd-Ellah, A. I. Awad, H. F. A. Hamed, and A. A. M. Khalaf, "Parallel Deep CNN Structure for Glioma Detection and Classification via Brain MRI Images," in 2019 31st International Conference on Microelectronics (ICM), 2019, pp. 304-307.

[23] M. K. Abd-Ellah, A. A. M. Khalaf, A. I. Awad, and H. F. A. Hamed, "TPUAR-Net: Two Parallel U-Net with Asymmetric Residual-Based Deep Convolutional Neural Network 
for Brain Tumor Segmentation," in Image Analysis and Recognition, Cham, 2019, pp. 106-116: Springer International Publishing.

[24] A. M. Ibraheem, K. H. Rahouma, and H. F. A. Hamed, "Automatic MRI Breast tumor Detection using Discrete Wavelet Transform and Support Vector Machines," in 2019 Novel Intelligent and Leading Emerging Sciences Conference (NILES), 2019, vol. 1, pp. 88-91.

[25] E. Bayoumi, M. Abd-Ellah, A. A. M. Khalaf, and R. Gharieb, "Brain Tumor Automatic Detection from MRI Images Using Transfer Learning Model with Deep Convolutional Neural Network," Journal of Advanced Engineering Trends, vol. 41, no. 2, 01/06 2022.

[26] D. Chicco and G. Jurman, "The advantages of the Matthews correlation coefficient (MCC) over F1 score and accuracy in binary classification evaluation," $B M C$ Genomics, vol. 21, no. 1, p. 6, 2020/01/02 2020. 